\title{
SmartGate - connecting wireless sensor nodes to the Internet
}

\author{
G. U. Gamm, S. Sester, and L. M. Reindl \\ Laboratory for Electrical Instrumentation, Department of Microsystems Engineering - IMTEK, \\ Georges-Koehler-Allee 106, 79110 Freiburg, Germany \\ Correspondence to: G. U. Gamm (gamm@imtek.de)
}

Received: 7 December 2012 - Revised: 17 April 2013 - Accepted: 18 April 2013 - Published: 3 May 2013

\begin{abstract}
Wireless sensor nodes inside buildings are used to read out sensor data and to control actuators. The nodes need to operate for a long time with a single battery. Often the sensor data should be accessible via Internet from every point of the world. When using a standard Wi-Fi connection, the battery of the node would be depleted after a few hours due to idle currents in receive state. Using sensor nodes with included wake-up receivers can prolong the lifetime of the sensor network to several years. However, no gateway exists that can, on the one hand, connect itself to the Internet and on the other hand can send out the special coded wake-up signal needed by the wake-up receivers on the nodes. In this work we want to bridge this gap by introducing the SmartGate. It is a gateway that has two transceivers incorporated on a single printed circuit board (PCB). A Wi-Fi module connects itself to an existing Wi-Fi network and listens for incoming messages. A CC430 microcontroller analyzes the incoming Wi-Fi messages and builds up the corresponding wake-up signal with included 16-bit address coding. The wake-up signal is sent out using the integrated CC1101 transceiver core from Texas Instruments. A woken-up node will read out its sensor data and will transmit it back to the gateway, where it will be packed into a TCP/IP packet and sent back to the user. The use of the gateway allows the implementation of a wireless sensor network with wake-up receivers that can be accessed via Internet from every point of the world.
\end{abstract}

\section{Introduction}

The Internet of things (IoT) implies the connection of ubiquitous embedded devices to the Internet. It is regarded as the third step in the evolution of information technology after the Internet and mobile communication. Applications of the IoT in logistics and environment can be found in Atzori et al. (2010). Examples of wireless sensor networks in health care can be found in De Boeck (2011) and Sjoland et al. (2012). For an increasing amount of applications, the connection of the WSN (wireless sensor network) to the Internet is an attractive feature. In the health care area, for example, it enables a doctor to check vital functions of a body area network of a patient using a computer from his/her office. Another example is the smart home, where the lights and shutters can be controlled via Internet from any point of the world. The applications in the area of IoT have two ap- parently contrary requirements. The first is the permanent accessibility of the nodes via Internet, which requires a turned on radio in listening mode and therefore results in a high current consumption. The second is a long lifetime of the sensor network of several years without changing the battery. One possible solution to these contradictions is the use of a sensor network with wake-up receivers. The wake-up receiver only consumes a few microwatts of energy while still being able to listen for a special coded wake-up signal. The nodes can then be triggered to active state with this signal at any time. Up till now, the wake-up signal could not be sent out directly from a Wi-Fi router. Therefore, a mediator is needed to translate the incoming TCP/IP requests into the appropriate wake-up signals for the nodes. This function is realized by our SmartGate. The term Wi-Fi refers in the following to a WLAN based on the 802.11 standard. The idea of using a central gateway to connect a WSN to the Internet is not 


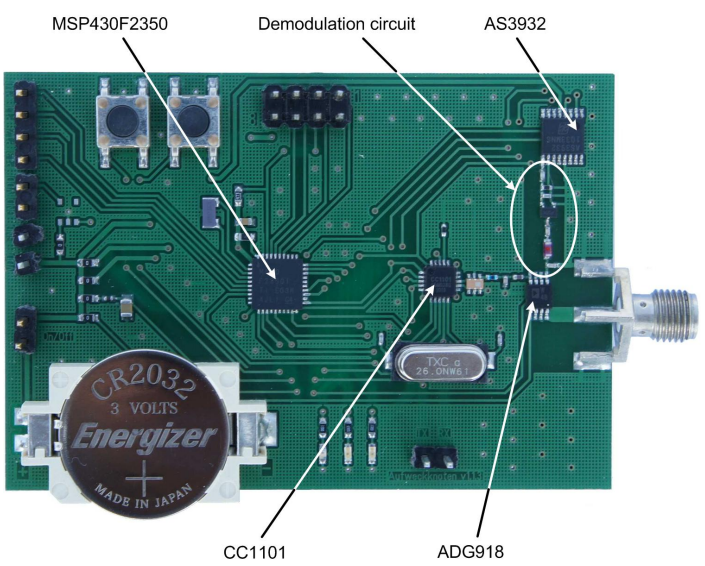

Figure 1. Photo of the assembled sensor node with wake-up receiver.

new. In Bian et al. (2011) a gateway for connecting ZigBeebased nodes to the Internet is presented. In Zhu et al. (2010) the gateway functionality is realized by a smartphone. Another example of IP-based WSNs and the additional gateway can be found in Hong et al. (2010) and Ha et al. (2012). However, in all of these examples, the sensor nodes have a permanent active channel or a duty-cycle-based rendezvous scheme. Therefore, a long lifetime in addition to permanent accessibility is not achieved.

The paper is organized in the following way. Section 2 gives a short description about the used wireless sensor nodes that can be targeted by the SmartGate. Section 3 explains the concept and the motivation of the SmartGate. In Sect. 3.1 the server system behind the SmartGate is introduced. Section 3.2 gives information about the hardware and software of the gateway. In Sect. 3.3 the wake-up signal, which is sent by the gateway, is explained. Measurements of the communication range and the current consumption can be found in Sect. 4, followed by the final conclusion.

\section{Sensor node with wake-up receiver}

The used sensor nodes with wake-up receiver that can be targeted by the SmartGate are presented and described in detail in Gamm et al. (2012). They feature a current consumption of $2.8 \mu \mathrm{A}$ in standby mode and a sensitivity for a wake-up signal of $-53.6 \mathrm{dBm}$ resulting in a wake-up range of $47 \mathrm{~m}$. To every node a 16-bit address can be assigned that enables a selective wake-up. In this case, the address information has to be integrated in the wake-up signal. Figure 1 shows a picture of the used nodes. An overview about existing wake-up technologies and their benefits can be found in Demirkol et al. (2009).

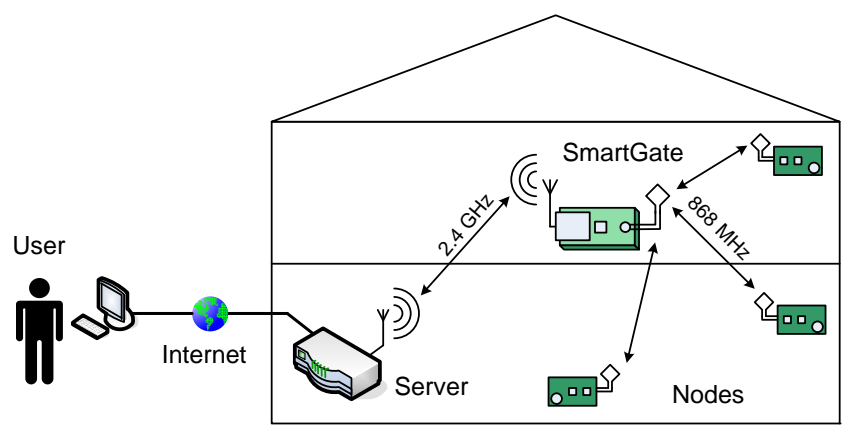

Figure 2. Whole system overview.

\section{SmartGate concept}

In comparison to the sensor nodes, the SmartGate is not required to be battery-powered. It can be placed at any point inside a building where a wired power supply is available. The optimum position for the gateway would be in the center of the wireless sensor network, so that the distance to every node is equal (assuming the same attenuation in all directions). For the communication with the nodes, the gateway uses the CC430F5137 system on chip in combination with the CC1190 RF front end (both from Texas Instruments). For the communication with the server, the gateway uses a WiFly RN-131C from Roving Networks. As the server we currently use a Shuttle XS35V2 personal computer with an integrated Intel NM10 Chipset for tethered and untethered communication. For better communication an external $2.4 \mathrm{GHz}$ half dipole omnidirectional antenna instead of the printed circuit board (PCB) antenna was used. The communication concept can be seen in Fig. 2.

A user from any point in the world can open a website with a graphical user interface for controlling the nodes. The website is hosted on the server that has a cable connection to the Internet. If the user requests the status of a node (e.g., inside a building), a TCP/IP packet will be sent to the server. The server spans a private Wi-Fi network inside the building and forwards the request to the SmartGate. The gateway extracts the command and the ID of the requested node and builds up a wake-up signal with the included node ID and sends it out. The woken up node will first turn on its main radio and will send back an acknowledgement to the SmartGate. The SmartGate will then send a message with the user's request. That can be the readout of a sensor or the control of an actuator. The node will accomplish the command and will send back the sensor data or the actuator status to the SmartGate. The SmartGate will send the message back to the server PC, and from there it will find the way back to the user via the Internet. A structured diagram of the message chain can be found in Fig. 3.

The domain from the user to the server makes use of the standard Internet infrastructure. The user can send his/her request from any computer or smartphone connected to the 

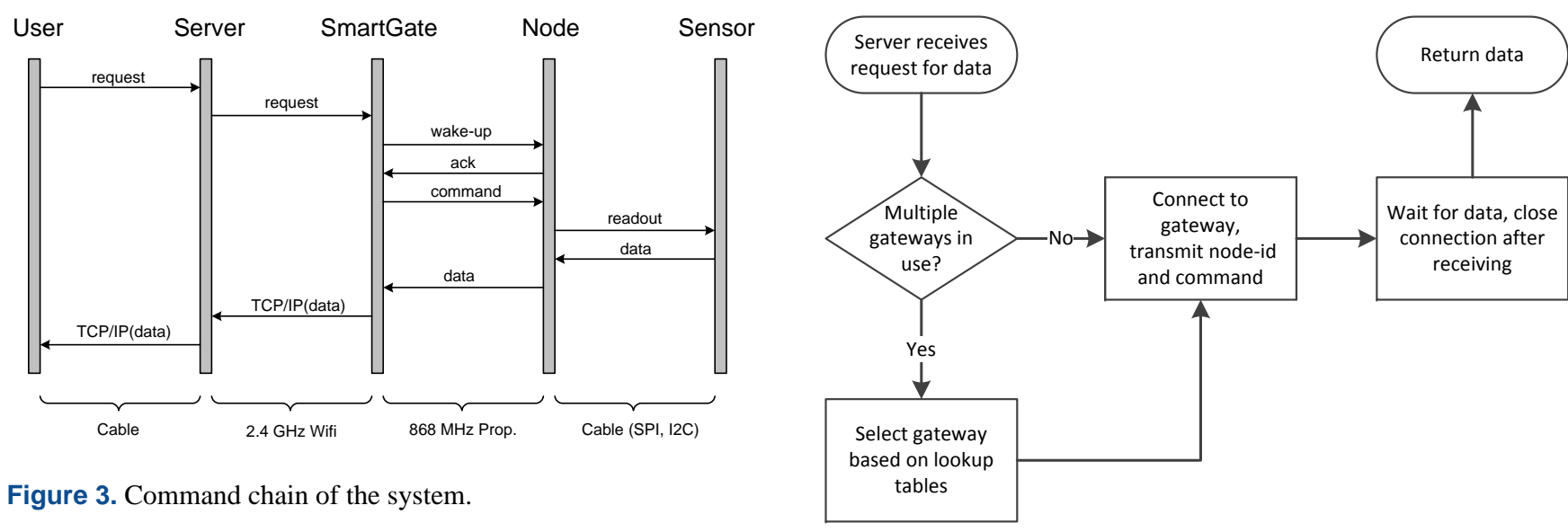

Figure 3. Command chain of the system.

Internet. The domain within the house from the server to the SmartGate uses a secured Wi-Fi network working at 2.4 GHz. The domain from SmartGate to the nodes uses the $868 \mathrm{MHz}$ ISM (industrial, scientific, and medical) band with a proprietary protocol. In this domain two kind of packages are sent: first, the wake-up packages consisting of the $125 \mathrm{kHz}$ signal modulated on the $868 \mathrm{MHz}$ carrier with included node ID and, second, normal data packages using GFSK modulation and data handling mechanism. The domain between node and sensor is dominated by cable connection with protocols like SPI or I2C.

\subsection{Server}

As a server a Shuttle XS35V2 personal computer running on Ubuntu 12.04 LTS is used. It connects to the Internet via Ethernet and works as an Wi-Fi access point by spanning a Wi-Fi network with hostapd ${ }^{1}$. Additionally, an Apache web server was used in combination with PHP 5.3 to provide both the user interface and the control software. The user interface is programmed with modern technologies such as HTML5 and JavaScript to provide access from normal desktop clients as well as from mobile clients such as smartphones or tablets. The control software is built entirely with PHP 5.3, which is a good choice regarding easiness of development as well as portability of the code. It consists of two parts: one part for the interaction with the client like evaluating given arguments and returning final data, and one for the communication with the SmartGate. Due to its architecture, it is possible to separate the two parts to allow direct access for other systems apart from the currently implemented user interface. An example would be to provide autonomous control by external systems. The current control software would then be used as a simple filter to regulate the access to the gateway, which should not be reachable directly from the Internet for security reasons. It is possible to integrate the server directly onto the SmartGate by using a tiny embedded web server. Since there might be more than one SmartGate installed in a build-

\footnotetext{
${ }^{1}$ http://w1.fi/hostapd/
}

Figure 4. Flowchart of the server software.

ing, we did stick to the concept of only one central server and one or more SmartGates communicating with it. A simplified flow chart of the server software can be seen in Fig. 4.

\subsection{Gateway}

The gateway uses the RN-131C from Roving Networks, which is a low cost solution for adding Wi-Fi compatibility to any system with an UART interface. It supports the IEEE $802.11 \mathrm{~b} / \mathrm{g}$ standards and features in its current firmware version 2.35 the most common authentication methods (WEP, WPA, WPA2) as well as most of the current protocols used in networks (DHCP, DNS, ARP, ICMP, TCP and UDP). The $\mathrm{RN}-131 \mathrm{C}$ connects to the Wi-Fi provided by the server and stays in standby mode, waiting for any incoming connections. Upon any new connection, the RN-131C forwards the received data to the CC430F5137 system on chip (microcontroller and transceiver on one die). The CC430F5137 evaluates the received data to eliminate the possibility of transmission errors. This is done by checking parity bytes and additional information such as the predetermined length of the incoming data against the real length. If the data are not correctly transmitted, they are truncated. By not getting any answer from the CC430 at all - despite having successfully opened the connection - the server can recognize that it has to resend the request due to a previous transmission failure. After having successfully verified the incoming data, the CC430 continues with enabling its own $868 \mathrm{MHz}$ radio transceiver. It then generates and sends the modulated signal for waking up the requested node and starts to listen for any incoming data. Within the next $300 \mathrm{~ms}$, the gateway will check any received data for being a valid response from the requested node. If it finds it is a valid response, it will forward it to the server. If it does not receive a valid response within the time, it could send a specific error message to the server. At the moment, the control software running on the server will retry to wake the node until - after a certain number of failed attempts - it 


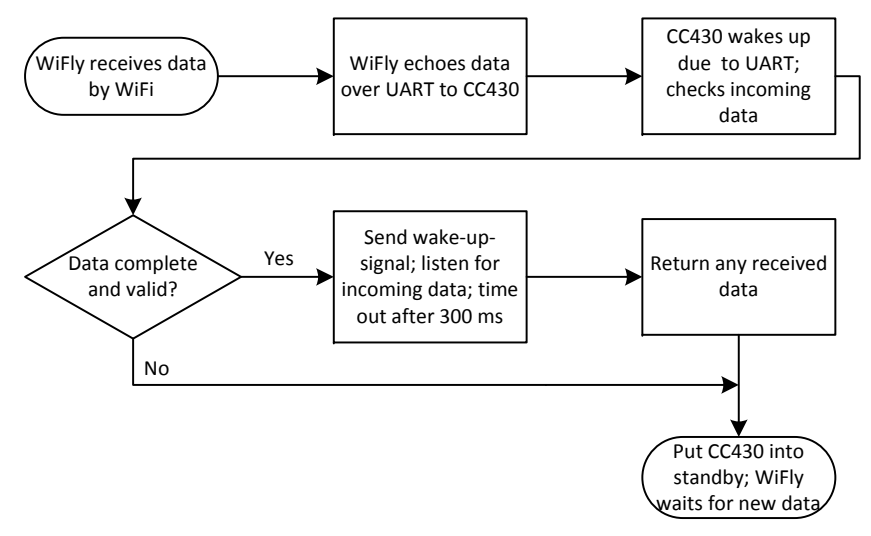

Figure 5. Flowchart of the gateway software.

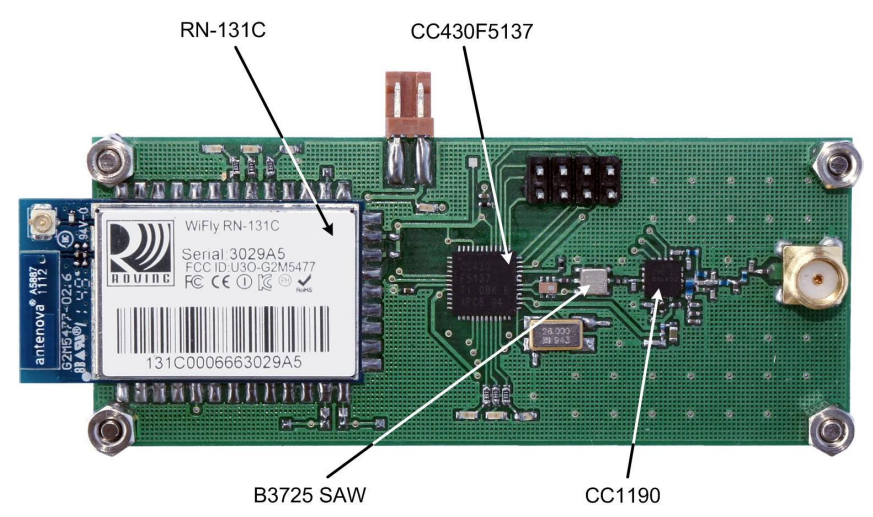

Figure 6. Photo of the assembled SmartGate.

is safe to assume that the node is offline. Either way, the gateway will close the connection afterwards, therefore telling the server that the transmission has finished. Figure 5 shows a flowchart of the gateway software.

The SmartGate is built on a $1 \mathrm{~mm}$ FR4 substrate. Besides the RN-131C Wi-Fi module and the CC430F5137, the board incorporates the CC1190 radio fronted from Texas Instruments. It allows increasing the sending power up to $+20 \mathrm{dBm}$, which results in a higher wake-up and communication range. Additional elements on the board are status light-emitting diodes (LEDs) for the Wi-Fi module and the CC430, a $26 \mathrm{MHz}$ crystal for the radio transceiver, a SAW filter from Epcos to suppress unwanted harmonics, JTAG interface and a chip balun from Johanson Technology. A photo of the assembled SmartGate can be seen in Fig. 6.

\subsection{Wake-up signal}

The expected wake-up signal by the nodes is composed of a carrier burst, preamble and address pattern as can be seen in Fig. 7.

To send out a valid wake-up signal, the SmartGate has to follow several steps. First, the $125 \mathrm{kHz}$ wake-up signal has to be built up whereby the correct 16-bit address informa-

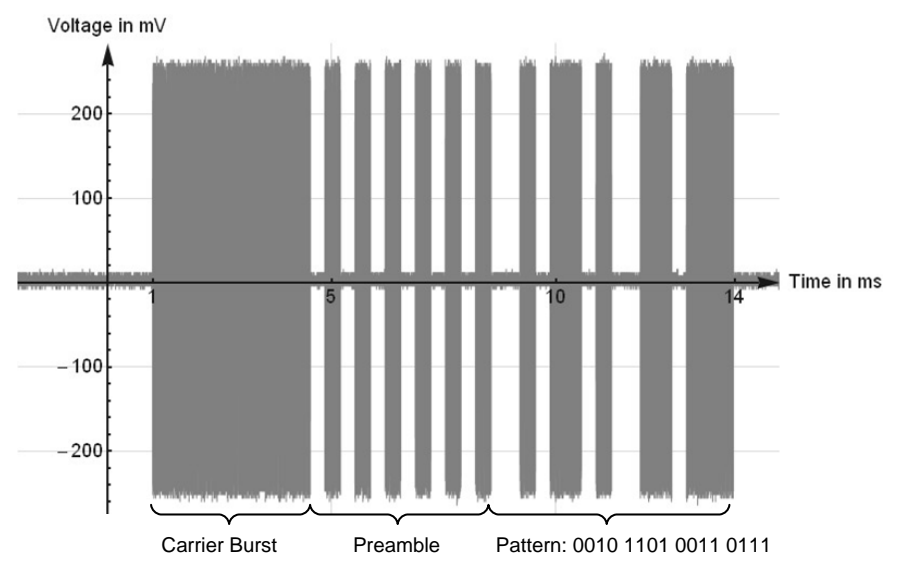

Figure 7. Expected wake-up signal by the sensor nodes.

tion has to be integrated. Second, the resulting signal must be modulated on the $868 \mathrm{MHz}$ carrier using OOK (on-off keying). And, third, the signal must be sent out using the infinite packet length mode of the $\mathrm{CC} 1101$ radio to overcome the limiting 64-byte FIFOs. Step one and two can be combined in the process of building the wake-up packet. Sending out a byte of 0xAA (binary 10101010) with a data rate of $250 \mathrm{kBit} \mathrm{s}^{-1}$ at a carrier frequency of $868 \mathrm{MHz}$ results in the modulation of two $125 \mathrm{kHz}$ square periods on the carrier. Using this scheme the whole wake-up packet with a length of 402 byte can be built up. The normal FIFO mode of the CC1101 radio only supports packet length up to 64 byte. To send out a packet with a length of 402 byte without interruption, the infinite packet length mode has to be used. It works by filling the FIFO initially with 64 byte. When these bytes are sent, an interruption can be used to monitor the amount of bytes still remaining in the FIFO. If 32 byte are sent, the interruption is triggered and 32 new bytes from the wake-up packet are filled on the fly in the FIFO. Therefore, the end of packet interruption of the $\mathrm{CC} 1101$ radio is first triggered when the last 32 byte from the wake-up packet are sent out.

\section{Measurements}

\subsection{Wi-Fi communication range}

In wireless devices one of the key properties is the communication range. The $\mathrm{RN}-131 \mathrm{C} \mathrm{Wi}-\mathrm{Fi}$ module communicates with a standard Wi-Fi network, in our case provided by the Shuttle XS35V2 PC. We tested the Wi-Fi communication range by successively moving the SmartGate away from the Shuttle PC while testing if it still could communicate with it. A Wi-Fi network connection is indicated by a green status LED. No Wi-Fi connection is indicated by a red LED. When using the internal PCB antenna of the RN-131C WiFly module on the SmartGate, a communication distance of $190 \mathrm{~m}$ was achieved. When connecting an external $2.4 \mathrm{GHz}$ dipole 


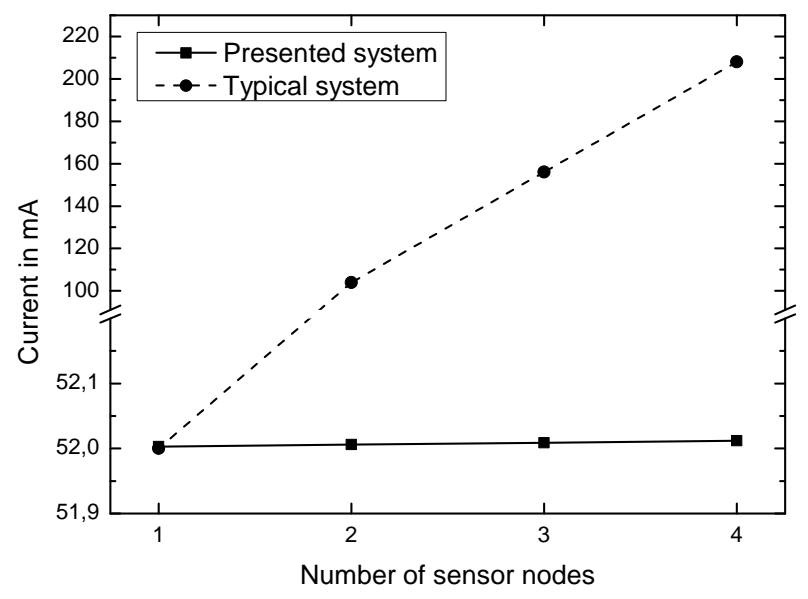

Figure 8. Comparison of a network with Wi-Fi nodes against a network with SmartGate and nodes with wake-up receivers.

antenna to the WiFly module, the communication range was extended up to $290 \mathrm{~m}$.

\subsection{Wake-up range}

The wake-up range from the SmartGate to a sleeping sensor node was done in an open air field. The SmartGate was programmed to send a coded wake-up signal in an infinite loop. One of the sensor nodes was programmed to wake up at the specific address and indicate the wake-up with a blinking LED. The SmartGate and the sensor node were mounted on poles of $1.2 \mathrm{~m}$ height each and were moved away from each other successively. The blinking, and therefore a positive wake-up, was measured up to a distance of $80 \mathrm{~m}$.

\subsection{Current consumption}

The current of the devices was measured using a digital multimeter 34401A from Agilent. The current consumption of the SmartGate in idle mode was measured to $52 \mathrm{~mA}$, while sending the wake-up signal with active amplifier front end the current was measured to $275 \mathrm{~mA}$. Let us look at a typical small sensor network consisting of four nodes that should be connected to the Internet. Integrating the WiFly module on every node results in a total current consumption of $220 \mathrm{~mA}$ in standby mode. Using four nodes with wake-up receivers and one SmartGate results in a current consumption of $52 \mathrm{~mA}$, dominated by the SmartGate. While adding additional nodes to the network will increase the current in a linear way for the typical system, it will add only $2.8 \mu \mathrm{A}$ in the presented system. Figure 8 compares the two systems against each other.

\section{Conclusions}

The SmartGate can be improved in three major points:

- Security: the CC430F5137 has a built-in AES hardware accelerator, which would allow fully encrypted communication with the control software of the server and with the sensor nodes. This could prevent attacks from malicious devices even when not used in its own Wi-Fi. This is especially important in scenarios where the nodes control actuators (e.g., doors or windows) and where an additional security layer is needed. Furthermore, the $\mathrm{RN}-131 \mathrm{C}$ can be programmed with a password that has to be sent upon any new connection. This would give a first rudimentary protection against attackers. Another possibility for an encrypted communication on the IPside would be the use of IPsec, which is a protocol suite for a secure IP communication.

- Scalability: while it is possible to use this system in large-scale environments, it was not explicitly improved for the use in such systems. Applications in this field should implement some kind of routing system for the gateway node communication, which allows the server to talk to a specific node without the need for a lookup table.

- Bidirectional communication: by now, the nodes are only contacted by the control software of the server. It is possible to change the control software as well as the gateway's configuration to support communication started by the node, which can be essential in use cases such as burglar alarm systems, where immediate reaction is necessary.

The SmartGate provides a practical solution for connecting a wireless sensor network that makes use of wake-up receivers to the Internet. For that task, the gateway can build up a coded wake-up signal with included address information and can send it out with an integrated $868 \mathrm{MHz}$ transceiver. It has the function of a mediator between the Internet with its TCP/IP protocol and the sensor network with its wake-up signals and proprietary data packets. The long wake-up range of $80 \mathrm{~m}$ enables a single SmartGate to cover the communication with nodes in a circular area with a diameter of $160 \mathrm{~m}$. The SmartGate represents a flexible and effective solution to access a network of wireless sensor nodes with wake-up receivers from the Internet.

Acknowledgements. This work has partly been supported by the German Research Foundation (DFG) within the Research Training Group 1103 (Embedded Microsystems).

Edited by: U. Schmid 


\section{References}

Atzori, L., Iera, A., and Morabito, G.: The Internet of Things: A survey, Comput. Netw., 54, 2787-2805, doi:10.1016/j.comnet.2010.05.010, 2010.

Bian, J., Fan, D., and Zhang, J.: The new intelligent home control system based on the dynamic and intelligent gateway, in: Broadband Network and Multimedia Technology (IC-BNMT), 2011 4th IEEE International Conference on, 526-530, 2011.

De Boeck, J.: Game-changing opportunities for wireless personal healthcare and lifestyle, in: Solid-State Circuits Conference Digest of Technical Papers (ISSCC), 2011 IEEE International, 1521, 2011.

Demirkol, I., Ersoy, C., and Onur, E.: Wake-up receivers for wireless sensor networks: benefits and challenges, Wireless Communications, IEEE, 16, 88-96, 2009.

Gamm, G. U., Kostic, M., Sippel, M., and Reindl, L. M.: Lowpower sensor node with addressable wake-up on-demand capability, Int. J. Sensor Networks, 11, 48-56, 2012.
Ha, M., Kim, S. H., Kim, H., Kwon, K., Giang, N., and Kim, D.: SNAIL gateway: Dual-mode wireless access points for WiFi and IP-based wireless sensor networks in the internet of things, in: Consumer Communications and Networking Conference (CCNC), 2012 IEEE, 169-173, 2012.

Hong, S., Kim, D., Ha, M., Bae, S., Park, S. J., Jung, W., and Kim, J.-E.: SNAIL: an IP-based wireless sensor network approach to the internet of things, Wireless Communications, IEEE, 17, 3442, 2010.

Sjoland, H., Anderson, J., Bryant, C., Chandra, R., Edfors, O., Johansson, A., Mazloum, N., Meraji, R., Nilsson, P., Radjen, D., Rodrigues, J., Sherazi, S., and Owall, V.: A Receiver Architecture for Devices in Wireless Body Area Networks, Emerging and Selected Topics in Circuits and Systems, IEEE Journal on, 2, 8295, 2012.

Zhu, Q., Wang, R., Chen, Q., Liu, Y., and Qin, W.: IOT Gateway: BridgingWireless Sensor Networks into Internet of Things, in: Embedded and Ubiquitous Computing (EUC), 2010 IEEE/IFIP 8th International Conference on, 347-352, 2010. 